\title{
Avaliação da temperatura retal em vacas leiteiras no pós-parto
}

\author{
Rectal temperatures in postpartum cows
}

\author{
Marion Burkhardt de Koivisto'; Katia Denise Bresciani²; César Esper³; Thais Mioto Martinelli ${ }^{4}$; \\ Leslie Cristina Scarpelli5; Silvia Helena Venturolli Perri²
}

\begin{abstract}
Resumo: O trabalho teve como objetivo correlacionar dados da parturição com o acompanhamento diário da temperatura retal no pós-parto imediato em fêmeas bovinas. Foram selecionadas aleatoriamente 180 vacas leiteiras provenientes de sete propriedades, em Araçatuba (SP) e região, sendo monitoradas durante o puerpério precoce, no período de setembro de 1999 a julho de 2000. Do primeiro ao décimo dia pós-parto a temperatura retal (TR) de todos os animais foi aferida no intervalo das 05:00 às 08:00 horas da manhã, utilizando-se termômetro eletrônico (M525 - GLA Agricultural Electronics, San Luis Obispo, CA 93401-7500). Foi considerada acima da normalidade TR superior a $39,5^{\circ} \mathrm{C}$, sendo colhidas informações sobre eventuais distocias, partos gemelares, retenção de membranas fetais, alteração do estado geral, ingestão de alimentos, produção de leite e descarga vaginal. Houve associação significativa entre descarga vaginal purulenta ou sanguinolenta e estado febril, sendo que dos 180 animais observados, 26 (14,4\%) apresentaram corrimento vaginal patológico, destes dez $(38,5 \%)$ tiveram febre, enquanto que os bovinos sem presença de secreção alterada tiveram elevação na temperatura corpórea em 17,5\% dos casos. Também ocorreu associação significativa entre parto distócico e placenta retida, 37 vacas $(20,6 \%)$ mostraram parturição difícil e, destas, oito (21,6\%) retiveram as membranas fetais, enquanto as fêmeas que pariram normalmente tiveram $4,2 \%$ (seis animais) de retenção de secundinas. Constatou-se que vacas apenas com retenção placentária não apresentaram sinais clínicos, exceto presença de uma transitória redução no apetite e da produção láctea. Este monitoramento diário, através da aferição da temperatura retal, consiste em importante instrumento adicional, podendo indicar precocemente alterações reprodutivas, como possíveis infecções uterinas, maximizando a produção leiteira e a performance reprodutiva futura.
\end{abstract} Palavras-chave: pós-parto, vacas leiteiras, temperatura retal.

\begin{abstract}
The purpose of this study was to evaluate parturition data with the rectal temperature in the early postpartum period of dairy cows. One hundred and eighty cows were randomly selected between September 1999 and July 2000 , in seven dairy farms located in the Northwest region of São Paulo, Brazil. For the first ten days postpartum, rectal temperature (RT) was taken between 5:00 and 8:00 a.m. using an electronic thermometer (M525 - GLA Agricultural Electronics, San Luis Obispo, CA 93401-7500). Cows with $\mathrm{RT}>39,5^{\circ} \mathrm{C}$ were enrolled and other signs like calving difficulties, dystocia due to twins, fetal membrane retention, ill appearance, off-feed, milk production and vaginal discharge were recorded. Significant association was observed between vaginal discharge and fever. Considering 180 animals, 26 (14,4\%) showed vaginal discharge, among them ten $(38,5 \%)$ were pyretic, while in cows without vaginal discharge only 27 $(17,5 \%)$ showed higher rectal temperatures. Difficult parturition and retained membranes also were statistically different, 37 cows $(20,6 \%)$ presented dystocia, among them eight $(21,6 \%)$ had retention of the afterbirth, while in the cows with normal parturition only six $(4,2 \%)$ showed retention of the fetal membranes. It was observed that cows with or without retained fetal membranes did not show clinical signs less a transitory reduction of appetite and milk production. This daily monitoring of rectal temperature represents an aditional tool indicating precociously reprodutive alterations, like uterine infections, maximizing milk production and future uterine performance.
\end{abstract}

Key words: postpartum, dairy cows, rectal temperature.

\section{Introdução}

Uma infinidade de sistemas de manejo tem demonstrado, ao longo do tempo, incremento na produtividade da indústria de produção leiteira. Contribuições visando à maximização da rentabilidade deste tipo de empresa rural constituem o mais amplo objetivo destes programas (PETERS e BALL, 1995).

Analisando detalhadamente o complexo conjunto de eventos inter-relacionados que desencadeiam elevação na produtividade e otimização da atividade leiteira, assim como a interação harmônica entre o desenvolvi-mento tecnológico e o aprimoramento genético, inseriu-se como fator essencial a manutenção da eficiência reprodutiva (KNUTTI et al., 2000).

Baseados em critérios de fertilidade estipulou-se um período médio de 40 a 45 dias para que se processem a regressão e a involução uterina ao estado normal (GARCIA e LARSSON, 1982). Cada dia de atraso, além de reduzir o intervalo ótimo entre a parição e a concep-

\footnotetext{
1 Departamento de Clínica, Cirurgia e Reprodução Animal /FOA/ UNESP, Araçatuba, S.P.

2 Departamento de Apoio, Produção e Saúde Animal /FOA/ UNESP, Araçatuba, S.P.

${ }^{3}$ Departamento de Reprodução Animal e Medicina Preventiva/FCAV/UNESP, Campus de Jaboticabal

${ }^{4}$ Graduanda do Curso de Medicina Veterinária/FOA/ UNESP, Araçatuba, S.P.

${ }^{5}$ CPPAR-Centro de Pesquisas em Sanidade Animal, FCAVJ/UNESP, Campus de Jaboticabal
} 
ção (em torno de 85 dias pós-parto) (NOAKES, 1991), tende a diminuir proporcionalmente a margem de lucratividade do criador. Uma fase especialmente estressante para vacas leiteiras acontece ao redor da parturição e no pós-parto imediato (UPHAM, 1996).

A medicina veterinária vem empregando o termômetro clínico como meio auxiliar no diagnóstico e controle do progresso de enfermidades em animais desde 1770 (WHITE, 1993).

Considerando a escassa informação bibliográfica nacional sobre monitoração da temperatura retal no período pós-parto imediato em vacas de leite, realizouse o presente trabalho com o objetivo de correlacionar dados como o histórico do parto, partos gemelares, distocias, retenção das membranas fetais, produção láctea, ingestão de alimentos e a presença de corrimento vaginal anômalo nos animais com o acompanhamento diário da temperatura retal.

\section{Material e Métodos}

Foram utilizadas 180 vacas leiteiras, selecionadas aleatoriamente, provenientes de sete propriedades do Município de Araçatuba, Estado de São Paulo e acompanhadas no período de setembro de 1999 a julho de 2000.

A temperatura retal das vacas recém-paridas foi aferida a partir do primeiro ao décimo dia pós-parto, das 05:00 às 08:00 horas da manhã, utilizando-se termômetro eletrônico (M525 - GLA Agricultural Electronics, San Luis Obispo, CA 93401-7500). Valores de temperatura retal entre 38,5 a $39,5^{\circ} \mathrm{C}$ são esperados para bovinos adultos hígidos na aferição por meio de termômetro retal (BLOOD e RADOSTIS; 1989; STÖBER, 1993), sendo este parâmetro adotado neste estudo.
Na ficha de observação diária, foram colhidas informações sobre eventuais distocias, retenção de placenta, alteração do estado geral, como anorexia, produção de leite e descarga vaginal (esta última notificada apenas quando observada com o aspecto de secreção purulenta, sanguinolenta ou com presença de debris celulares).

A análise estatística foi elaborada com a utilização do teste Qui-quadrado ou teste exato de Fisher com o intuito de verificar associação entre variáveis qualitativas. O teste t de Student e o teste de Mann-Whitney foram usados para comparar médias de dois grupos independentes, para as variáveis produção láctea e o percentual de perda de peso, respectivamente.

\section{Resultados e Discussão}

Das 180 vacas acompanhadas, $143(79,4 \%)$ apresentaram parto eutócico e 37 (20,6\%) tiveram parturição distócica. Entre as fêmeas que pariram normalmente, $27(18,9 \%)$ apresentaram febre e $116(81,1 \%)$ não evidenciaram esta síndrome. Por sua vez, dos 37 animais com distocia, dez (27\%) estiveram febris e 27 (73\%) não mostraram elevação na temperatura corporal. Não houve associação significativa entre parto distócico e febre (Tabela 1).

Estabeleceu-se para o delivramento fisiológico um período médio de até 12 horas pós-parto ou abortamento, e após esse prazo, houve interferência na descarga de lóquios e, consequentemente, da eliminação da contaminação bacteriana (SMITH, 1990; NOAKES, 1991; TONIOLLO e VICENTE, 1995; ARTHUR, 1996).

Em nosso trabalho, constatou-se que vacas apenas com retenção placentária não apresentaram sinais clínicos, exceto pela presença de uma transitória redução no apetite e da produção láctea. Também não

Tabela 1 - Distribuição do número $(\mathrm{N})$ e porcentagem $(\%)$ dos animais segundo a ocorrência de hipertermia associada ao tipo de parto, descarga vaginal e placenta retida.

\begin{tabular}{|c|c|c|c|c|c|c|c|c|}
\hline \multirow{3}{*}{ Variável } & \multirow{3}{*}{ Categoria } & \multicolumn{4}{|c|}{ Temperatura retal } & \multirow{3}{*}{$\begin{array}{l}\text { Total } \\
\mathbf{N}\end{array}$} & \multirow[b]{3}{*}{$\%$} & \multirow{3}{*}{$\mathbf{P}$} \\
\hline & & \multicolumn{2}{|c|}{ Hipertermia } & \multicolumn{2}{|c|}{ Normotermia } & & & \\
\hline & & $\mathbf{N}$ & $\%$ & $\mathbf{N}$ & $\%$ & & & \\
\hline \multirow{3}{*}{ Tipo de parto } & Eutócico & 27 & 18,9 & 116 & 81,1 & 143 & 100,0 & \multirow{3}{*}{$0,274^{(1)}$} \\
\hline & Distócico & 10 & 27,0 & 27 & 73,0 & 37 & 100,0 & \\
\hline & Total & 37 & 20,6 & 143 & 79,4 & 180 & 100,0 & \\
\hline \multirow{3}{*}{ Placenta retida } & Ausência & 34 & 20,5 & 132 & 79,5 & 166 & 100,0 & \multirow{3}{*}{$1,000^{(2)}$} \\
\hline & Presença & 3 & 21,4 & 11 & 78,6 & 14 & 100,0 & \\
\hline & Total & 37 & 20,6 & 143 & 79,4 & 180 & 100,0 & \\
\hline \multirow{3}{*}{$\begin{array}{c}\text { Descarga } \\
\text { vaginal }\end{array}$} & Ausência & 27 & 17,5 & 127 & 82,5 & 154 & 100,0 & \multirow{3}{*}{$0,015^{(1)}$} \\
\hline & Presença & 10 & 38,5 & 16 & 61,5 & 26 & 100,0 & \\
\hline & Total & 37 & 20,6 & 143 & 79,4 & 180 & 100,0 & \\
\hline
\end{tabular}

${ }^{(1)}$ nível descritivo do teste Qui-quadrado ${ }^{(2)}$ nível descritivo do teste exato de Fisher 
ocorreu associação significativa entre estado febril e placenta retida. Do total de 180 bovinos monitorados, $166(92,2 \%)$ não tiveram retenção placentária e em somente 14 deles $(7,8 \%)$ não foi notada liberação das membranas fetais (Tabela 2). Verificou-se também que somente três $(21,4 \%)$ dos 14 animais que tiveram retenção das secundinas manifestaram estado febril (Tabela 1). Na Nova Zelândia, a não-expulsão das membranas fetais foi vista numa proporção de 1,96\%, em 36 vacas leiteiras mantidas em regime de pastagens, num período de um ano (ROBERTS, 1979).

$A$ retenção placentária é freqüentemente seguida de uma involução mais demorada do útero (ARTHUR et al., 1996), como também pode haver maior falha na gestação subseqüente (LOPEZ-GATIUS, et al., 1996). Em alguns casos, não houve diferenças na performance reprodutiva entre vacas com ou sem essa patologia puerperal (KANEKO et al., 1997).

Por outro lado, levantamento efetuado em 3022 vacas prenhas lactentes, na Espanha, demonstrou que medidas de redução do risco de retenção placentária e piometra devem aumentar o índice de concepção em vacas de leite (LÓPEZ-GATIUS et al., 1996), alcançando uma melhor performance reprodutiva (FOURICHON et al., 2000; ROCHE et al., 2000).

Neste estudo averiguou-se uma ocorrência de descarga vaginal patológica de 13,2\% (22 animais) e 28,6\% (quatro animais), respectivamente, nos animais sem (166) e com (14) retenção placentária. Não foi estabelecida associação significativa entre descarga vaginal alterada e placenta retida. Através do exame externo do animal, esta inflamação genital não pode ser notada, a menos que exista corrimento vaginal ou fluidos uterinos ressecados na cauda ou na região perineal (GRUNERT e GRUNERT, 1990). Dos 26 animais com descarga vaginal, dez $(38,5 \%)$ evidenciaram estado febril, ao mesmo tempo entre as 154 vacas sem corrimento, apenas $27(17,5 \%)$ tiveram febre, mostrando associação significativa entre descarga vaginal e febre (Tabela 2).

Tabela 2 - Distribuição do número (N) e porcentagem (\%) dos animais segundo o tipo de parto associado com placenta retida e descarga vaginal.

\begin{tabular}{|c|c|c|c|c|c|c|c|c|}
\hline \multirow{3}{*}{ Variável } & \multirow{3}{*}{$\begin{array}{c}\text { Placenta } \\
\text { retida }\end{array}$} & \multicolumn{4}{|c|}{ Descarga vaginal } & \multirow{2}{*}{\multicolumn{2}{|c|}{ Total }} & \multirow{3}{*}{$\mathbf{P}$} \\
\hline & & \multicolumn{2}{|c|}{ Ausência } & \multicolumn{2}{|c|}{ Presença } & & & \\
\hline & & $\mathbf{N}$ & $\%$ & $\mathbf{N}$ & $\%$ & $\mathbf{N}$ & $\%$ & \\
\hline \multirow{3}{*}{ Placenta retida } & Ausência & 144 & 86,8 & 22 & 13,2 & 166 & 100,0 & \multirow{3}{*}{0,124} \\
\hline & Presença & 10 & 71,4 & 4 & 28,6 & 14 & 100,0 & \\
\hline & Total & 154 & 85,6 & 26 & 14,4 & 180 & 100,0 & \\
\hline
\end{tabular}

$\mathrm{P}=$ nível descritivo do teste exato de Fisher

Entre as entidades capazes de ocasionar retenção das membranas fetais, todas relevantes, incluem-se parto prematuro, podendo estar associada a aborto infeccioso, secção cesariana e indução farmacológica da parturição (PETERS e BALL, 1995).

Também ocorreu associação significativa entre placenta retida e parto difícil. Enquanto 37 animais com distocia $(21,6 \%)$ retiveram as secundinas, em parições eutócicas, apenas seis (4,2\%) fêmeas tiveram retenção das membranas fetais (Tabela 2). A ocorrência de retenção placentária subseqüente a partos eutócicos não assistidos foi relatada na ordem de oito a 11\% (ARTHUR, 1996).

Diversos autores têm reconhecido que vacas com distocia, retenção da placenta, gêmeos, fetos mortos e vários problemas metabólicos desenvolvem mais comumente metrite que outras vacas (MORROW, 1986, GRUNERT e BIRGEL, 1989).

$\mathrm{Na}$ Tabela 3 constata-se que 143 vacas tiveram parto normal, 126 (88,1\%) não apresentaram e 17 (11,9\%) evidenciaram descarga vaginal. Entre as vacas que tiveram distocia, houve secreção vaginal em nove ani- mais $(24,3 \%)$, tendo sido notada uma tendência à associação entre corrimento vaginal e parto distócico $(p=0,055)$.

A infecção uterina pós-parto foi tida como a causa predominante da infertilidade bovina (ERB et al., 1981 a, b) e, independentemente da causa, a endometrite deve ser tratada o mais rapidamente possível, caso se deseje manter um intervalo curto entre a parturição e ciclos estrais férteis (STUDER e MORROW, 1978). Foi descrito também, que metrite puerperal aguda apresentava uma incidência em torno de 10,7\% a 36,4\% (BARLETT, 1986; STEVENSON, 1988).

No que concerne às vacas com parturição complicada, observamos uma redução percentual do peso inicial de $3,5 \%$ em relação ao peso final nos dez primeiros dias pós-parto, enquanto se constatou uma diminuição ponderal de $1,8 \%$ no grupo que pariu normalmente, sendo esta diferença considerada estatisticamente significativa $(p=0,0295)$.

A produção média de leite do grupo que não teve placenta retida (148 animais) foi em média 15,2 $\pm 6,41$, não diferindo estatisticamente $(p=0,1735)$ do grupo 
Tabela 3 - Distribuição do número $(\mathrm{N})$ e porcentagem (\%) dos animais segundo o tipo de parto associado com placenta retida e descarga vaginal.

\begin{tabular}{|c|c|c|c|c|c|c|c|c|}
\hline \multirow{3}{*}{ Variável } & \multirow{3}{*}{ Categoria } & \multicolumn{4}{|c|}{ Tipo de parto } & \multirow{2}{*}{\multicolumn{2}{|c|}{ Total }} & \multirow{3}{*}{$\mathbf{P}$} \\
\hline & & \multicolumn{2}{|c|}{ Eutócico } & \multicolumn{2}{|c|}{ Distócico } & & & \\
\hline & & $\mathbf{N}$ & $\%$ & $\mathbf{N}$ & $\%$ & $\mathbf{N}$ & $\%$ & \\
\hline \multirow{3}{*}{$\begin{array}{c}\text { Descarga } \\
\text { vaginal }\end{array}$} & Ausência & 126 & 88,1 & 28 & 75,7 & 154 & 85,6 & \multirow{3}{*}{$0,055^{(1)}$} \\
\hline & Presença & 17 & 11,9 & 9 & 24,3 & 26 & 14,4 & \\
\hline & Total & 143 & 100,0 & 37 & 100,0 & 180 & 100,0 & \\
\hline \multirow{3}{*}{ Placenta retida } & Ausência & 137 & 95,8 & 29 & 78,4 & 166 & 92,2 & \multirow{3}{*}{$0,002^{(2)}$} \\
\hline & Presença & 6 & 4,2 & 8 & 21,6 & 14 & 7,8 & \\
\hline & Total & 143 & 100,0 & 37 & 100,0 & 180 & 100,0 & \\
\hline
\end{tabular}

${ }^{(1)}$ nível descritivo do teste Qui-quadrado ${ }^{(2)}$ nível descritivo do teste exato de Fisher

que reteve a placenta $(12,8 \pm 4,95)$. Já foi demonstrado que infecções uterinas (LEWIS, 1997), distocia e retenção placentária contribuíram para redução da produção de leite em vacas (RAJALA-SCHUZ e GRÖHN, 1998).

Tanto o parto distócico, como a infecção uterina, foram estados patológicos que elevaram o risco de descarte das vacas afetadas, tanto no momento da sua ocorrência como no final da lactação (RAJALA-SCHULTZ e GRÖHN, 1999 a, b, c).

Vacas com puerpério patológico foram acometidas posteriormente por uma moderada a severa metrite (GRÖHN et al.,1990; SMITH, 1990; GRÖHN et al., 1995, a,b; LEWIS, 1997), tendo esta inflamação capacidade de acarretar menor taxa de concepção (KNUTTI, et al., 2000).

Animais com metrite séptica demonstraram descarga vaginal evidente, diminuição da produção de leite, em alguns casos laminite, anorexia, febre e depressão, devido a toxemia (SMITH, 1990).
O efeito da condição corporal na eficiência reprodutiva de vacas de leite foi estudado e observou-se que, reduzindo-se a porcentagem do rebanho de vacas com baixa condição corporal, aumentava a eficiência reprodutiva (MOREIRA et al., 2000).

Vacas primíparas com condição corpórea fraca ao parto tem um período anovulatório longo (TONIOLLO e VICENTE, 1995; NATION et al., 2000). Pesquisadores israelenses (MARKUSFELD e EZRA, 1993) correlacionaram dados de condição corpórea e metrite, com a performance da primeira lactação em vacas holandesas e analisaram a inter-relação entre alterações reprodutivas, produção láctea, reprodução e manejo do rebanho para a elaboração de um esquema terapêutico e de manejo (GRÖHN e RAJALA-SCHULTZ, 2000).

\section{Conclusão}

Concluímos que o monitoramento diário através da aferição da temperatura retal em vacas leiteiras, nos 
primeiros dez dias pós-parto, consiste em importante instrumento adicional, podendo indicar precocemente alterações reprodutivas, como possíveis infecções uterinas.

\section{Referências Bibliográficas}

ARTHUR, G.H. Veterinary reproduction and obstetrics, London: Saunders, 1996. 726p.

BARLETT, P.C. et al.. Metritis complex in Michigan HolsteinFriesian cattle: incidence, descriptive epidemiology and estimated economic impact. Preventive Veterinary Medicine, Amsterdam, v.4, p.235-248, 1986.

BLOOD, D.C., RADOSTITIS, O.M. Veterinary Medicine. 7. ed. London: Baillieri Tindal, 1989. p.14-15.

ERB, H.N.; MARTIN, S.W.; ISON, N. Interrelationships between production and reproductive disease in Holstein cows: conditional relationships between production and disease. Journal Dairy Science, Champaign, v. 64, p. 272281, 1981a.

ERB, H.N.; MARTIN, S.W.; ISON, N. Interrelationships between production and reproductive disease in Holstein Cows. Path. Analysis. Journal Dairy Science, Champaign, v. 64, p. $282-289,1981$ b.

FOURICHON, C.; SEEGERS, H.; MALHER, X. Effect of disease on reproduction in the dairy cow: a Meta-analysis. Theriogenology, Stoneham, v.53, n.9, p.1729-1759, 2000.

GARCIA.; LARSSON, K. Clinical findings in post-partum dairy cows. Nordisk Veterinaermedicin, Copenhagen, v.34, n.7, p. 255-263, 1982.

GRÖHN, Y.T.; EICKER, S.W.; HERTL, J.A. The association between previous 305-day milk yield and disease in New York State dairy cows. Journal Dairy Science, Champaign, v.78, p.1693-1772, 1995a.

GRÖHN, Y.T. et al.. The effect of disease on culling Holstein dairy cows in the New York State. Journal Dairy Science, Champaign, v.78, p.1693-1702, 1995b.

GRÖHN, Y.T. et al.. Epidemiology of reproductive disorders in dairy cattle: associations among host characteristics, disease and production. Preventive Veterinary Medicine, Amsterdam, v. 8, p. 25-39, 1990.

GRÖHN, Y.T.; RAJALA-SCHULTZ, P.J. Epidemiology of reproductive performance in dairy cows. Animal Reproduction Science, Amsterdam, v.60-61, p.605-614, 2000.

GRUNERT, E., BIRGEL, E.H. Obstetrícia veterinária. 3.ed. Porto Alegre: Sulina, 1989. 323p.

GRUNERT, E.; GRUNERT, D. The problems of the sucess of afterbirth removal in cattle. Expert veterinary opinion. Tierarztl Prax, v.18, n.5, p.473-476, 1990.

KANEKO, K. et al.. Effect of retained placenta on subsequent bacteriological and cytological intrauterine environment and reproduction in Holstein dairy cows. Theriogenology, Stoneham, v.48, n.4, p.617-624, 1997.

KNUTTI, B.; KUPFER, U.; BUSATO, A. Reproductive efficiency of cows with endometritis after treatment with intrauterine infusions or prostaglandin injections, or no treatment. Journal of Veterinary Medicine: series A Physiology Pathology Clinical Medicine. Berlin, v.47, n.10, p.609-615, 2000.
LEWIS, G.S. Uterine health and disorders. Journal Dairy Science, Champaign, v.80, n. 5, p.984-994, 1997.

LÓPEZ-GATIUS, F. et al.. Effect of reproductive disorders previous to conception on pregnancy attrition in dairy cows. Theriogenology, Stoneham, v.46, n.4, p.643-648, 1996.

MARKUSFELD, O.; EZRA, E. Body measurements, metritis, and postpartum performance of first lactation cows. Journal Dairy Science, Champaign, v.76, n.12, p.3771-3777, 1993.

MOREIRA, F. et al.. Effect of body condition on reproductive efficiency of lactating dairy cows receiving a timed insemination. Theriogenology, Stoneham, v.53, n.6, p.13051319, 2000.

MORROW, D.A. Current therapy in Theriogenology 2: diagnosis, treatment and prevention of reproductive diseases in small and large animals. Philadelphia: W.B. Saunders, 1986. 1143p.

NATION, D.P. et al.. Hormonal and ovarian responses to a 5 -day progesterone treatment in anoestrus dairy cows in the third week post-partum. Animal Reproduction Science, Amsterdam, v.63, n. 1-2, p. 13-25, 2000.

NOAKES, D.E. Fertilidade e obstetrícia em bovinos. São Paulo: Livraria Varela, 1991. 139p.

PETERS, A.R.; BALL, P.J.H. Reproduction in cattle. $2^{\text {nd }}$ ed. Oxford: Blackwell Science. 1995. 234p

GEORGHIOU, G. P., TAYLOR, C. E. Culling of dairy cows: Part I. Effects of diseases on culling in finish Ayrshire cows. Preventive Veterinary Medicine, Amsterdam, v. 41, p. 195208, 1999a.

GEORGHIOU, G. P., TAYLOR, C. E. Culling of dairy cows: Part II. Effects of diseases and reproductive performance on culling in Finnish Ayrshire cows. Preventive Veterinary Medicine, Amsterdam, v. 41, p. 279-94, 1999b. 